\title{
Duration of CS termination delay in avoidance extinction following partial and continuous reinforcement
}

\author{
PETER F. GALVANI \\ State University of New York, College at Brockport, Brockport, New York 14420
}

\begin{abstract}
Mongolian gerbils received either continuous or partial (50\%) reinforcement in acquisition of a shuttle avoidance response $(\mathrm{Ra})$. In extinction, separate groups received $\mathrm{CS}$ termination at one of three delay intervals, 0,5 , or $10 \mathrm{sec}$, combined with shock omission. Removal of prompt CS offset produced rapid extinction of the $\mathrm{Ra}$ for both partial and continuous reinforcement animals. However, partially reinforced gerbils subjected to 5 -sec delayed CS offset demonstrated a precipitous loss in Ra strength relative to their continuously reinforced counterparts. These results were discussed in terms of the interaction between the efficacy of the CS termination contingency in extinction and acquisition reinforcement schedule.
\end{abstract}

Although delay of CS termination following response completion has long been known to produce inferior avoidance acquisition relative to immediate CS termination (e. g., Kamin, 1956; Mowrer \& Lamoreaux, 1942), the introduction of delayed CS termination has been employed as an extinction procedure only more recently. Thus, Owen (1963) demonstrated that delaying CS offset for $20 \mathrm{sec}$ following an avoidance response (Ra) in extinction greatly decreased $\mathrm{Ra}$ strength compared to prompt CS termination. Katzev (1967) subsequently found that the ease of weakening the Ra varied directly as a function of delay duration $(0-10 \mathrm{sec})$ following an Ra.

Both Owen and Katzev omitted shock in extinction. The present author (Galvani, 1973a), on the other hand, assessed the effects of the CS termination contingency, following both partial and continuous reinforcement, in extinction in which shocks were unavoidable. These results showed that partial reinforcement (PR) in avoidance acquisition, in which nonreinforced trials involved the elimination of both CS termination and US avoidance, considerably enhanced the efficacy of the CS termination contingency in $\mathrm{Ra}$ maintenance. Specifically, a comparison of the relative strengths of association ( $\omega^{2}$ statistic, see Hays, 1963, pp. 381-384) between delay of CS termination ( 0 vs. $5 \mathrm{sec}$ ) and extinction performance, following either PR or continuous reinforcement (CR), showed that this relationship was much stronger in the case of PR-trained animals. Galvani (1973a) speculated that the enhanced reinforcing capacity of CS termination following intermittent reinforcement might be attributable to its unique informational role, i. e., CS termination was the only reliable feedback signaling a successful $\mathrm{Ra}$ the animals experienced under the PR schedule.

The research reported in the present paper was supported by Grant B04355-001 awarded to the author by the National Science Foundation.
The present study was undertaken to further evaluate the effects of duration of CS termination delay on extinction performance following both continuous and intermittent reinforcement. Shock was omitted in extinction, since it is possible that the unavoidable-shock extinction procedures in Galvani (1973a) attenuated the effectiveness of CS termination. Assuming PRavoidance training enhances the degree of control of the CS termination contingency, one might expect a steeper delay of CS termination (reinforcement) gradient in extinction following PR training, since for a given CS delay the loss in reinforcement for the $\mathrm{Ra}$ should be greater in PR-trained compared to CR animals.

\section{Subjects \\ METHOD \\ The subjects were 60 adult (4-to 6-month-old) male gerbils obtained from Tumblebrook Farms, West Brookfield, Massachu- setts. All subjects were maintained on ad-lib food and water and housed in a continuously illuminated room.}

\section{Apparatus}

Two Scientific Prototype Model A100S toggle-floor shuttleboxes $(13.5$ by 13 by $40 \mathrm{~cm})$, described in detail in Galvani (1971), were employed. Each shuttlebox was enclosed in a ventilated sound-attenuated chamber illuminated by a $7.5-\mathrm{W}$ incandescent lamp. Both the continuous masking noise and the tonal CS $(2,500 \mathrm{~Hz})$ were delivered through an $8-\mathrm{cm}$ speaker centered above the shuttlebox via a BRS-Foringer AU-902 audio generator. The $C S$ raised the sound level $(85 \mathrm{~dB}$ with masking noise and ventilating fan on) approximately $2 \mathrm{~dB}$ on the $\mathrm{C}$ scale of a General Radio Type 1561-A sound-level meter. The US was a $.5-\mathrm{mA} .5$-sec scrambled electric shock delivered to the grid floor of the shuttlebox via a Grason-Stadler GS700 shock generator. Programming and data collection were accomplished by electromechanical equipment situated next to the soundattenuated chambers.

\section{Procedure}

Following a 10 -min adaptation period in the shuttleboxes, all animals received 100 acquisition trials, followed immediately by 100 extinction trials, at an average ITI of $30 \mathrm{sec}$. The design was a 2 by 3 factorial ( $n=10$ per group), in which one variable 

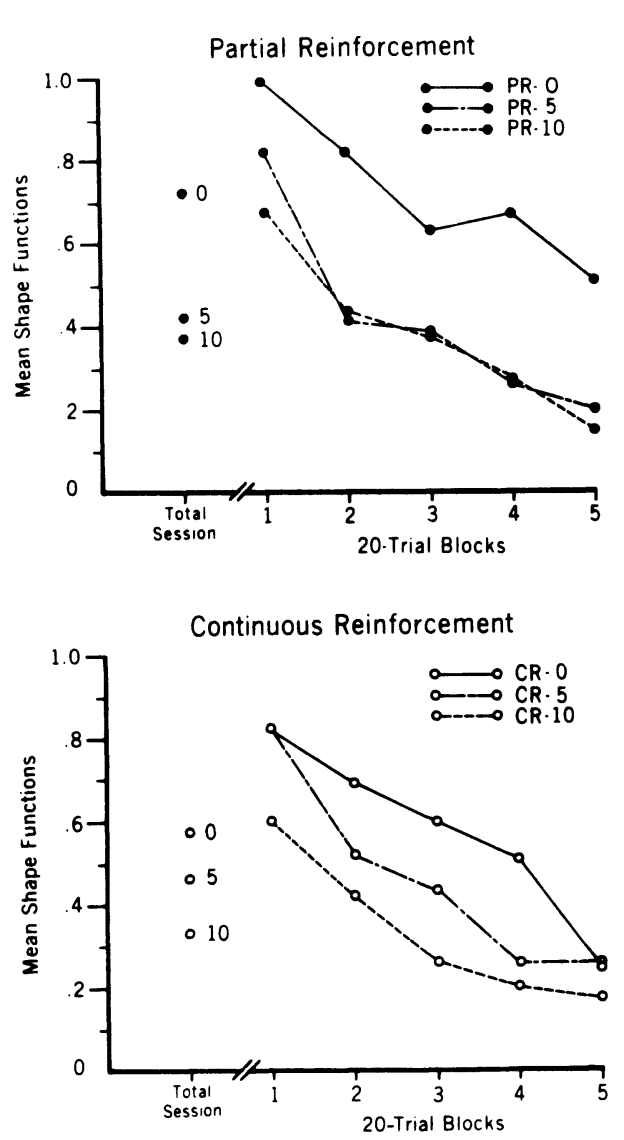

Figure 1. Mean shape functions for partial reinforcement (upper panel) and continuous reinforcement (lower panel) animals in extinction as a function of CS termination delay. Mean shape functions for total 100-trial extinction session are depicted to the left on each graph (parameter is CS delay interval in seconds), while mean shape functions for each group over five 20-trial blocks in extinction are shown to the right.

was reinforcement schedule in acquisition (PR vs. $C R$ ) and the second variable was CS termination delay in extinction $(0,5$, and $10 \mathrm{sec})$. Groups were labeled by reinforcement schedule and delay interval as follows: CR-0, CR-5, CR-10; PR-0, PR-5, PR-10.

The CS-US interval in acquisition was $5 \mathrm{sec}$, with shock presentation coinciding with CS offset. A reinforced trial was one on which a shuttle response ( $\mathrm{Ra}$ ) during the CS terminated the tone immediately and precluded shock. On nonreinforced trials, the CS remained on for $5 \mathrm{sec}$ and was inevitably followed by shock, irrespective of $\mathrm{Ra}$ occurrence. PR gerbils received a random $50 \%$ reinforced trials. If no $\mathrm{Ra}$ occurred on an acquisition trial, the 5 -sec CS was followed by the .5 -sec US for all subjects. On extinction trials, on the other hand, if no $\mathrm{Ra}$ occurred the CS terminated automatically after $5 \mathrm{sec}$. No shocks were presented in extinction. Duration of the CS delay interval was measured from the time of the initial $\mathrm{Ra}$ occurrence on a particular trial, regardless of length of Ra latency.

\section{RESULTS AND DISCUSSION}

The mean number of Ras in acquisition for $C R$ and PR animals was 61.8 and 49.8 , respectively. These means differed reliably, $\mathrm{F}(1,46)=6.22, \mathrm{p}<.05$, as did the mean number of Ras on the final 10 acquisition trials:
$\mathrm{CR}=8.4, \mathrm{PR}=5.9, \mathrm{~F}(1,46)=26.59, \mathrm{p}<.001$. These results are consistent with previous evidence that $P R$ gerbils are inferior in shuttlebox avoidance acquisition relative to CR gerbils, e. g., Galvani, 1973a.

Due to the reliable group differences obtained between PR and CR animals in acquisition, in order to evaluate relative degree of extinction between groups, the shape-function method of "correction" for initial acquisition differences (see Anderson, 1963, p. 165, Equation 4) was applied to each subject's extinction scores. Since the constant used to represent asymptotic extinction performance was estimated to be zero, the equation for transforming these data reduced to the number of Ras for each subject in a given 20-trial block in extinction, divided by the initial extinction level (estimated from each animal's terminal acquisition performance).

Figure 1 presents the mean shape functions for all six groups over 20-trial blocks in extinction. Also, the mean shape function for the total 100-trial extinction session is depicted for each group in Figure 1. Inspection of this figure clearly shows that all groups demonstrated a decrease in $\mathrm{Ra}$ strength in extinction, i. e., shape functions $<1$. Moreover, the loss of $\mathrm{Ra}$ strength increased progressively over trial blocks. An ANOVA performed on the mean shape functions of Figure 1 revealed that only the main effects of Delay of CS termination, $\mathrm{F}(2,42)=7.06, \mathrm{p}<.01$, and Trial Blocks, $\mathrm{F}(4,168)=53.73, \mathrm{p}<.001$, were significant. All other main effects and interactions were nonsignificant (all Fs $\leqslant 1.22$ ). Collapsing across trial blocks, follow-up $t$ tests showed that Group PR-0 was superior in resistance to extinction to both PR-5, $\mathrm{t}(14)=2.60, \mathrm{p}<.05$, and PR-10, $t(14)=2.47, p<.05$, but Groups PR-5 and PR-10 did not differ, $t<1$. On the other hand, the only significant pairwise comparison involving CR groups was between Groups CR-0 and CR-10, $\mathrm{t}(14)=2.21$, $p<.05$.

The effects of delayed CS termination, combined with shock omission, obtained in the present experiment are generally consistent with results obtained with other rodent species in both shuttlebox (Katzev, 1967; Owen, 1963) and one-way avoidance (Delprato, 1969) tasks. Thus, removal of the CS termination contingency produced rapid and reliable extinction of the Ra. Moreover, the greater the duration of CS delay following an $\mathrm{Ra}$ in extinction, the greater the relative loss in Ra strength.

On the other hand, the relationship between CS termination delay and acquisition reinforcement schedule was less clear. The prediction of a steeper gradient relating duration of CS delay and $\mathrm{Ra}$ persistence, following $P R$ relative to $C R$ training, was not verified by a Reinforcement Schedule by Delay Duration interaction. Pairwise group comparisons involving different delay intervals, however, revealed that PR gerbils exposed to a 5-sec delay showed considerable loss in Ra strength compared to PR animals that received 
prompt CS offset (in fact, PR-5 subjects showed as great a loss in Ra strength as PR-10 animals), while the comparable CR groups, CR-0 and CR-5, did not differ reliably in resistance to extinction. If loss of prompt CS termination in extinction produces a greater relative decrement in resistance to extinction following PR compared to $\mathrm{CR}$, then these results are consistent with previous evidence (Galvani, 1973a) that PR in avoidance acquisition enhances the efficacy of the CS termination contingency. Of course, the above effect was obtained only with the intermediate CS termination delay $(5 \mathrm{sec})$, not with the longer interval employed $(10 \mathrm{sec})$.

Another outcome of interest in the present study was the failure to obtain a partial reinforcement effect (PRE). In other words, the relative resistance to extinction of PR animals was not reliably greater than $C R$ gerbils. Other recent studies of intermittent reinforcement in shuttlebox avoidance learning with gerbils have not reported a PRE (Galvani, 1976; Galvani, Twitty, \& Foster, 1975) when shocks were omitted in extinction. The most compelling evidence of a PRE following avoidance learning has in fact been obtained in those studies in which shock was unavoidable on each extinction trial (Galvani, 1973a, b) although one exception to the above pattern exists (see Galvani, 1971). Thus, a PRE following shuttlebox avoidance learning in gerbils is apparently more readily observed when all shocks are unavoidable as opposed to the procedure of complete shock omission. Although the determinants of this pattern of results remain to be explored, taken together these results imply that the CS termination contingency is relatively more effective following PR than CR in perseverating Ras during extinction with shock unavoidable than during extinction without shock (cf. Bolles, 1975, p. 346).

\section{REFERENCES}

ANDERson, N. H. Comparison of different populations: Resistance to extinction and transfer. Psychological Review, 1963, 70. 162-179.

Bolles, R. C. Theory of motivation. 2nd. ed. New York: Harper and Row, 1975.

Delprato, D. J. Extinction of one-way avoidance and delayed warning-signal termination. Journal of Experimental Psychology, 1969, 80, 192-193.

Galvani, P. F. The effects of partial reinforcement on the acquisition and extinction of avoidance behavior in gerbils. Psychonomic Science, 1971, 24, 242-244.

Galvani, P. F. Effects of US-presentation and CS-termination contingencies on avoidance extinction following partial and continuous reinforcement. Learning and Motivation, 1973, 4, 197-206. (a)

Galvani, P. F. Punished and classical-trial extinction of avoidance in gerbils following partial and continuous reinforcement. Psychological Reports, 1973, 32, 723-729. (b)

Galvani, P. F. Effects of percentage of shock avoidance on avoidance behavior in gerbils. Bulletin of the Psychonomic Society, 1976, 7, 548-550.

Galvani, P. F., Twitty, M. T., \& Foster, K. M. Intermittent reinforcement and intertrial interval effects on shuttlebox avoidance in the gerbil. Bulletin of the Psychonomic Society, $1975,5,225-227$.

HaYs, W. L. Statistics for psychologists. New York: Holt, Rinehart and Winston, 1963.

KAMIN, L. J. The effects of termination of the CS and avoidance of the US on avoidance learning. Journal of Comparative and Physiological Psychology, 1956, 49, 420-424.

KATZEv, R. D. Extinguishing avoidance responses as a function of delayed warning signal termination. Journal of Experimental Psychology, 1967, 75, 339-344.

Mowrer, O. H., \& Lamoreaux, R. R. Avoidance conditioning and signal duration: A study of secondary motivation and reward. Psychological Monographs, 1942, 54 (No. 247).

OwEN, S. The effect on avoidance response extinction in rats of CS continuation and emotional constitution. Journal of Genetic Psychology, 1963, 103, 147-151.

(Received for publication May 13, 1976.) 